\title{
ATIVIDADE INIBITÓRIA DE PESTICIDAS PIRETROIDES SOBRE A GLUTATIONA S-TRANSFERASE
}

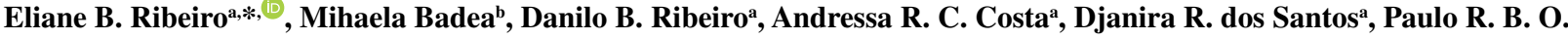 \\ Marques $^{\text {a }}$ G Gilvanda S. Nunes ${ }^{\mathrm{a}}$ \\ aDepartamento de Tecnologia Química, Universidade Federal do Maranhão, 65080-805 São Luís - MA, Brasil \\ bDepartment of Fundamental, Prophylactic and Clinical Specialties, Transilvania University of Brasov, 500039 Brasov, Romania
}

Recebido em 29/05/2021; aceito em 14/07/2021; publicado na web em 10/08/2021

\begin{abstract}
INHIBITORY ACTIVITY OF PYRETHROID PESTICIDES ON GLUTATHIONE S-TRANSFERASE. Glutathione S-transferase (GST) is one of the enzymes used to evaluate the effects caused by environmental contaminants. The aim of this work was to study the effects of four pyrethroid compounds (allethrin, cypermethrin, deltamethrin and fenpropathrin) on the catalytic activity of GST and to estimate the potential for environmental contamination in silico of these insecticides. Spectrophotometric analyses showed that the activity of the GST enzyme was inhibited by the four pesticides, with the values for the inhibition constant $\left(\mathrm{K}_{\mathrm{i}}\right)$ varying from 0.12 to $20 \mu \mathrm{mol} \mathrm{L}{ }^{-1}$, with the following order of inhibition from the highest to the lowest $\mathrm{K}_{\mathrm{i}}$ value: deltamethrin > cypermethrin > allethrin > fenpropathrin. They presented a competitive and non-competitive inhibition mechanism depending on the compound. The $\mathrm{IC}_{50}$ values (concentration that inhibits $50 \%$ of the enzyme activity) showed the insecticide deltamethrin with greater inhibitory capacity, since it reduced the activity of the GST enzyme by half, even though it was in a lower concentration in relation to the other analyzed compounds. The in silico evaluation indicated the possibility of most of the insecticides contaminating the surface waters (rivers and lakes) adhered to the suspended sediment, but did not show any potential for contamination of groundwater through leaching.
\end{abstract}

Keywords: synthetic pesticides; enzymatic activity; spectrophotometry; in silico; environmental contamination.

\section{INTRODUÇÃo}

Pesticidas são produtos químicos usados na agricultura para prevenir ou controlar os efeitos negativos de organismos nocivos. ${ }^{1}$ Conforme a sua finalidade podem ser classificados como inseticidas, fungicidas, herbicidas, rodenticidas, nematicidas, reguladores de crescimento de plantas e outros. ${ }^{2}$ Entre os pesticidas, estão os piretroides cujo desenvolvimento ocorreu a partir do piretrum, que é uma mistura de substâncias obtidas da maceração da flor do crisântemo (gênero Chrysanthemum spp.), conhecida por seus efeitos repelentes a insetos. Após estabelecidas as rotas para a sua síntese em 1980, surgiu a classe dos inseticidas piretroides. ${ }^{3}$

A partir desse período, foi desenvolvida uma variedade de estruturas piretroides, cujas formulações são empregadas em diversas áreas, no campo da veterinária, como antiparasitários em rebanhos, na medicina, como antiparasitários em humanos e em questões de saúde pública (combatendo mosquitos vetores de doenças) e no controle de pragas domésticas e agrícolas. ${ }^{4}$

Os piretroides constituem uma classe de inseticidas utilizados mundialmente, respondendo por mais de $17 \%$ do mercado global de agroquímicos. ${ }^{5}$ Devido ao crescente uso desses produtos, uma série de transtornos e modificações para o meio ambiente têm sido relatadas, como a contaminação do solo e de corpos hídricos, ${ }^{6}$ contaminação de alimentos, ${ }^{7,8}$ além da toxicidade para artrópodes aquáticos, abelhas e peixes. ${ }^{9}$

Apesar de serem conhecidos os impactos causados por pesticidas, ainda se faz necessário examinar os efeitos de compostos piretroides, sobretudo em relação a cinética da glutationa S-transferase (GST), que é uma enzima encontrada em vários seres vivos como fungos, plantas, animais e algumas bactérias. ${ }^{10}$ A GST constitui uma grande família de isoenzimas diméricas, citosólicas, que exercem função relevante na biotransformação e desintoxicação de compostos eletrofílicos (endógeno e exógeno como pesticidas e outros poluentes ambientais)

*e-mail: elianeribeiro.biologa@gmail.com e na defesa das células contra o estresse oxidativo nos organismos. ${ }^{11,12}$ Especificamente, nas reações de desintoxicação, a GST realiza a catálise da conjugação entre um xenobiótico e a glutationa reduzida (GSH), formando um composto conjugado, que é metabolizado ou excretado em uma etapa posterior. ${ }^{13}$

Nesse contexto, diversos estudos indicam que a GST é uma importante enzima que está entre os principais marcadores bioquímicos utilizados para avaliar a exposição e os efeitos provocados por contaminantes ambientais. ${ }^{14-16}$ Assim, este trabalho tem como finalidade investigar de forma in vitro, a inibição da atividade catalítica da glutationa $\mathrm{S}$-transferase usando os pesticidas piretroides aletrina, cipermetrina, deltametrina e fenpropatrina, demonstrados na Figura 1, bem como avaliar por meio in silico, o potencial de contaminação ambiental desses compostos.

\section{PARTE EXPERIMENTAL}

\section{Reagentes e soluções}

Foram adquiridos da Sigma-Aldrich os seguintes produtos: a enzima glutationa S-transferase (GST) de fígado equino, 1-cloro2,4-dinitrobenzeno (CDNB), glutationa reduzida (GSH), fosfato de potássio monobásico $\left(\mathrm{KH}_{2} \mathrm{PO}_{4}\right)$, ácido etilenodiamino tetra-acético - EDTA, etanol absoluto $\left(\mathrm{C}_{2} \mathrm{H}_{6} \mathrm{O}\right)$ e acetonitrila $\left(\mathrm{C}_{2} \mathrm{H}_{3} \mathrm{~N}\right)$. Os padrões analíticos foram adquiridos da Accu Standard (aletrina, deltametrina e fenpropatrina) e da Fluka (cipermetrina).

Para o preparo da solução tampão $(\mathrm{pH} 6,5)$ foram utilizados: fosfato de potássio monobásico, ácido etilenodiamino tetra-acético - EDTA e água deionizada purificada pelo sistema Milli-Q ${ }^{\circledR}$. A dissolução da GSH foi feita com tampão fosfato, e o CDNB, com etanol absoluto (70\% do volume) e água deionizada, sendo ambas preparadas antes de cada ensaio experimental. A solução estoque de GST foi preparada solubilizando-se em $1 \mathrm{~mL}$ de tampão fosfato (pH 6,5), $10 \mathrm{mg}$ dessa enzima em pó contida no frasco, sendo 25 unidades de enzima por miligrama, conforme informações do 




Aletrina $\left(\mathrm{C}_{19} \mathrm{H}_{26} \mathrm{O}_{3}\right)$

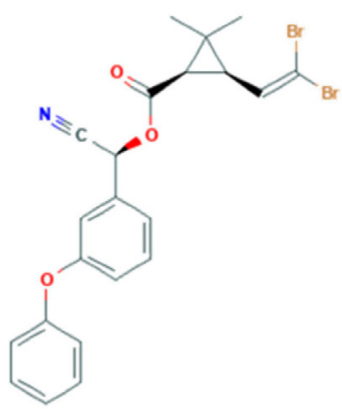

Deltametrina $\left(\mathrm{C}_{22} \mathrm{H}_{19} \mathrm{Br}_{2} \mathrm{NO}_{3}\right)$

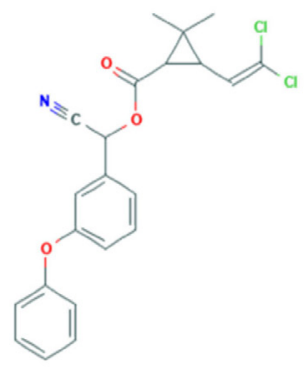

Cipermetrina $\left(\mathrm{C}_{22} \mathrm{H}_{19} \mathrm{Cl}_{2} \mathrm{NO}_{3}\right)$

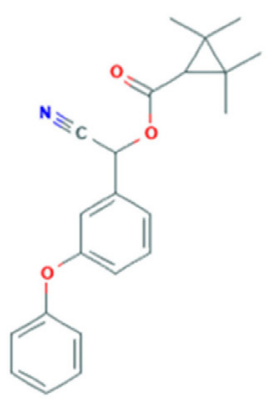

Fenpropatrina $\left(\mathrm{C}_{22} \mathrm{H}_{23} \mathrm{NO}_{3}\right)$
Figura 1. Estrutura química dos inseticidas piretroides analisados. Fonte: Pubchem

fabricante e foi armazenada a $-20^{\circ} \mathrm{C}$. Os padrões de pesticidas foram dissolvidos em acetonitrila. As soluções de trabalho foram preparadas em tampão, em concentração apropriada, a partir da solução-estoque.

\section{Equipamentos}

As medidas de absorbância foram registradas em espectrofotômetro UV/Vis (Thermoscientific, Orion AquaMate 8000) utilizando cubeta de quartzo com volume de $1 \mathrm{~mL}$ e $1 \mathrm{~cm}$ de caminho óptico.

\section{Determinação da atividade cinética da GST}

Para encontrar as melhores condições dos parâmetros a serem empregados nos ensaios de cinética enzimática, foi realizado um planejamento fatorial com três fatores e dois níveis, por meio do programa Minitab Statistical ${ }^{\circledR}$ versão 13.0. Os fatores correspondem aos reagentes empregados e os níveis à concentração mínima e máxima, desses reagentes (Tabela 1), as quais foram escolhidas tendo como base a realização de testes preliminares in vitro.

As variáveis foram as concentrações dos substratos 1-cloro-2,4dinitrobenzeno (CDNB) e glutationa reduzida (GSH), bem como a quantidade de enzima glutationa S-transferase (GST). Foram realizados oito ensaios em triplicata, tendo sido o experimento inteiramente casual.

A atividade da GST foi avaliada empregando-se o método já descrito na literatura ${ }^{17}$ adicionando-se quantidades variadas dos reagentes GSH e CDNB, em tampão fosfato (PBS) pH 6,5, em presença ou ausência da enzima GST, perfazendo um volume total de $450 \mu \mathrm{L}$ em cubeta de quartzo. A reação foi monitorada espectrofotometricamente, em um comprimento de onda de $340 \mathrm{~nm}$, por um tempo de 3 minutos. Uma unidade de GST representa a quantidade de enzima necessária para conjugar $1 \mu$ mol de CDNB com GSH por minuto.

Os parâmetros cinéticos, constante de Michaelis-Menten $\left(\mathrm{K}_{\mathrm{M}}\right)$ e a velocidade máxima $\left(\mathrm{V}_{\max }\right)$ foram calculados a partir da equação de Lineweaver-Burk (Equação 1). Sendo: $V_{o}=$ velocidade inicial da reação; $\mathrm{V}_{\max }=$ velocidade máxima de reação; $[\mathrm{S}]$ = concentração do substrato; e $\mathrm{K}_{\mathrm{M}}=$ constante de Michaelis-Menten. Para essa finalidade, foram realizados ensaios, mantendo-se fixa a carga enzimática da GST, a concentração de GSH permaneceu fixa e variou-se a concentração do CDNB, e o contrário, em que se fixou a concentração de CDNB e se variou a concentração de GSH. Serviu como controle o experimento realizado sem adição dos reagentes CDNB e GSH, conforme o ensaio.

$$
\frac{1}{V_{0}}=\left(\frac{K_{M}}{V_{\max }}\right) \cdot\left(\frac{1}{[S]}\right)+\frac{1}{V_{\max }}
$$

\section{Ensaio de inibição da enzima GST por inseticidas piretroides}

Nos testes para obtenção do mecanismo de inibição e constante de inibição- $K_{\mathrm{i}}$, foram empregadas concentrações fixas de $0,25 \mathrm{mg} \mathrm{L}^{-1}$ de cada pesticida em estudo, concentrações fixas de GSH, em tampão fosfato $0,1 \mathrm{~mol} \mathrm{~L}^{-1} \mathrm{pH}$ 6,5 com concentrações crescentes de CDNB em cubeta de quartzo. Os resultados foram representados conforme método Lineweaver-Burk (representação de duplo recíproco), plotando-se gráficos $1 / \mathrm{V}_{0}$ versus $1 /[\mathrm{S}]$.

Para o cálculo da concentração que inibe $50 \%$ da atividade da enzima - $\mathrm{IC}_{50}$, foram realizados ensaios com diferentes concentrações $\left(0,0625\right.$ a $\left.5 \mathrm{mg} \mathrm{L}^{-1}\right)$ de cada pesticida piretroide em concentração fixa do substrato CDNB, GSH e carga enzimática.

\section{Análise in silico do risco de contaminação aquática por pesticidas piretroides}

Essa etapa foi baseada na aplicação de duas metodologias: a aplicação dos critérios do método de GOSS ${ }^{18}$ para avaliação do risco de contaminação de águas superficiais e os critérios do índice de GUS - Groundwater Ubiquity Score,${ }^{19}$ para o risco de contaminação de águas subterrâneas.

No método de GOSS, são propostos critérios físico-químicos para cada contaminante, cujos valores são apresentados em intervalos matemáticos, o que resulta na classificação dos ingredientes ativos dos pesticidas conforme o potencial de contaminação associado ao sedimento ou dissolvido em água (Tabela 2).

No índice de GUS, utiliza-se, para o cálculo, os valores correspondentes as propriedades físico-químicas empregadas (meia-vida $\left(\mathrm{DT}_{50}\right)$ e o coeficiente de adsorção ao solo $\left(\mathrm{K}_{\mathrm{oc}}\right)$ ), de acordo com a Equação 2. Esse índice estabelece três

Tabela 1. Fatores e níveis estudados no planejamento fatorial $2^{3}$ para determinação cinética da atividade da enzima GST

\begin{tabular}{lcc}
\hline & & \\
Fatores & Mínimo & Níveis \\
\cline { 2 - 3 } & & $0,5 \mathrm{mmol} \mathrm{L}^{-1}$ \\
Glutationa reduzida - GSH & $0,2 \mathrm{mmol} \mathrm{L}^{-1}$ & $7,0 \mathrm{mmol} \mathrm{L}^{-1}$ \\
1-cloro-2,4-dinitrobenzeno - CDNB & $0,0125 \mathrm{U} \mathrm{mL}^{-1}$ & $2,0 \mathrm{mmol} \mathrm{L}^{-1}$ \\
Glutationa S- transferase - GST & $0,25 \mathrm{U} \mathrm{mL}^{-1}$ \\
\hline
\end{tabular}

Fatores = reagentes; Níveis = concentração dos reagentes; Concentração: GSH, CDNB, GST; U = atividade específica. 
Tabela 2. Potencial de transporte dos inseticidas piretroides de acordo com o Método de Goss

\begin{tabular}{lccc}
\hline \multirow{2}{*}{ Potencial } & \multicolumn{3}{c}{ Transporte associado ao sedimento } \\
\cline { 2 - 4 } & $\mathrm{DT}_{50}$ solo & $\mathrm{K}_{\mathrm{oc}}\left(\mathrm{mL} \mathrm{g}^{-1}\right)$ & $\begin{array}{c}\text { Solubilidade em } \\
\text { água }\left(\mathrm{mL} \mathrm{g}^{-1}\right)\end{array}$ \\
\hline \multirow{2}{*}{ Alto } & $\geq 40$ & $\geq 1000$ & - \\
& $\geq 40$ & $\geq 500$ & $\leq 0,5$ \\
\hline \multirow{2}{*}{ Baixo } & $<1$ & - & - \\
& $\leq 2$ & $\leq 500$ & - \\
& $\leq 4$ & $\leq 900$ & $\geq 0,5$ \\
Potencial & $\leq 40$ & $\leq 500$ & $\geq 0,5$ \\
& $\leq 40$ & $\leq 900$ & $\geq 2$ \\
\hline \multirow{2}{*}{ Alto } & & Transporte dissolvido em água \\
\hline & $\mathrm{DT}_{50}$ solo & $\mathrm{K}_{\mathrm{oc}}\left(\mathrm{mL} \mathrm{g}^{-1}\right)$ & Solubilidade em \\
& $>35$ & $<100000$ & $\geq 1$ \\
& $<35$ & $\leq 700$ & $\geq 10 \mathrm{e} \leq 100$ \\
\hline Baixo & - & $\geq 100000$ & - \\
& $\leq 1$ & $\geq 1000$ & - \\
\hline
\end{tabular}

$\mathrm{DT}_{50}=$ tempo de meia vida do pesticida; $\mathrm{K}_{\mathrm{oc}}=$ constante de adsorção à matéria orgânica; $\mathrm{S}=$ solubilidade em água. Fonte: adaptado de GOSS. ${ }^{18}$

faixas de classificação à tendência de lixiviação do pesticida: GUS $<1,8=$ não sofre lixiviação; $1,8<$ GUS $<2,8=$ faixa de transição; GUS > 2,8 = provável lixiviação.

$$
\text { GUS }=\log \left(\mathrm{DT}_{50 \text { soil }}\right) \cdot\left(4-\log \mathrm{K}_{\mathrm{oc}}\right)
$$

As informações sobre as propriedades físico-químicas dos compostos analisados foram adquiridas a partir de levantamento feito em artigos científicos relacionados e em banco de dados de acesso livre: Pesticide Properties Data (PPDB) e PubChem (pubchem.ncbi. nlm.nih.gov/).

\section{RESULTADOS E DISCUSSÃO}

\section{Análise quimiométrica: otimização do ensaio espectrofotométrico}

Os dados obtidos com o planejamento fatorial geraram diversos gráficos, apresentados a seguir. $\mathrm{O}$ gráfico de Pareto indicou que os parâmetros $\mathrm{C}$ (carga da enzima) e $\mathrm{B}$ (concentração de CDNB) foram os mais significativos para a resposta do sistema enzimático, podendo provocar variações mais expressivas, mesmo com pequenas diferenças em seus níveis (Figura 2).

A partir dessa análise, pode se afirmar que a concentração do substrato GSH não representou um parâmetro determinante, sugerindo que se forem utilizados níveis de concentração mais elevados $\left(7 \mathrm{mmol} \mathrm{L}^{-1}\right)$ ou menos elevados $\left(0,5 \mathrm{mmol} \mathrm{L}^{-1}\right)$, não implicaria em diferença significativa na resposta cinética.

A Figura 3a apresenta o gráfico de superfície, o qual indica que, para se alcançar os valores de absorbância entre 0,3 e 0,4 (ideal para início dos trabalhos envolvendo inibição da enzima), deve-se trabalhar com o parâmetro carga de enzima em um nível mais baixo. Já para o parâmetro concentração do CDNB, pode ser tanto o nível alto como baixo de carga enzimática, pois a variação dele não afetou o sistema quando a concentração de GSH foi fixada no menor nível $\left(0,5 \mathrm{mmol} \mathrm{L}^{-1}\right)$.

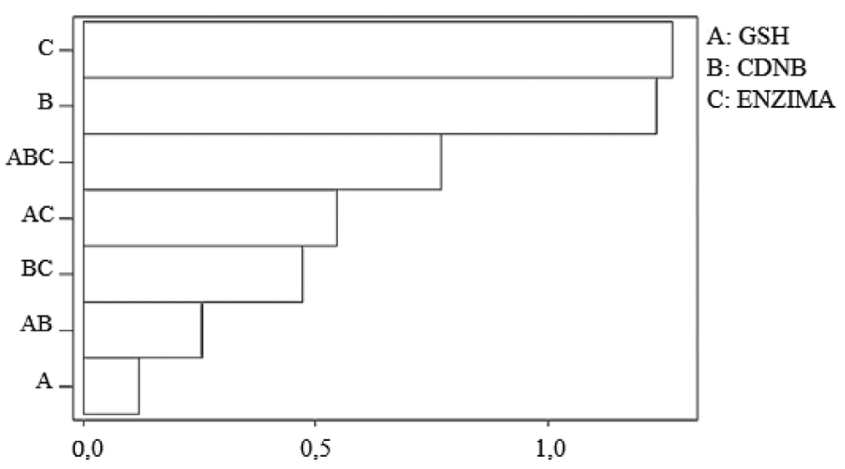

Figura 2. Gráfico de Pareto representando a influência das concentrações dos substratos 1-cloro-2,4-dinitrobenzeno (CDNB) e glutationa reduzida (GSH) e a carga da enzima glutationa $S$-transferase (GST), nos resultados cinéticos

a)
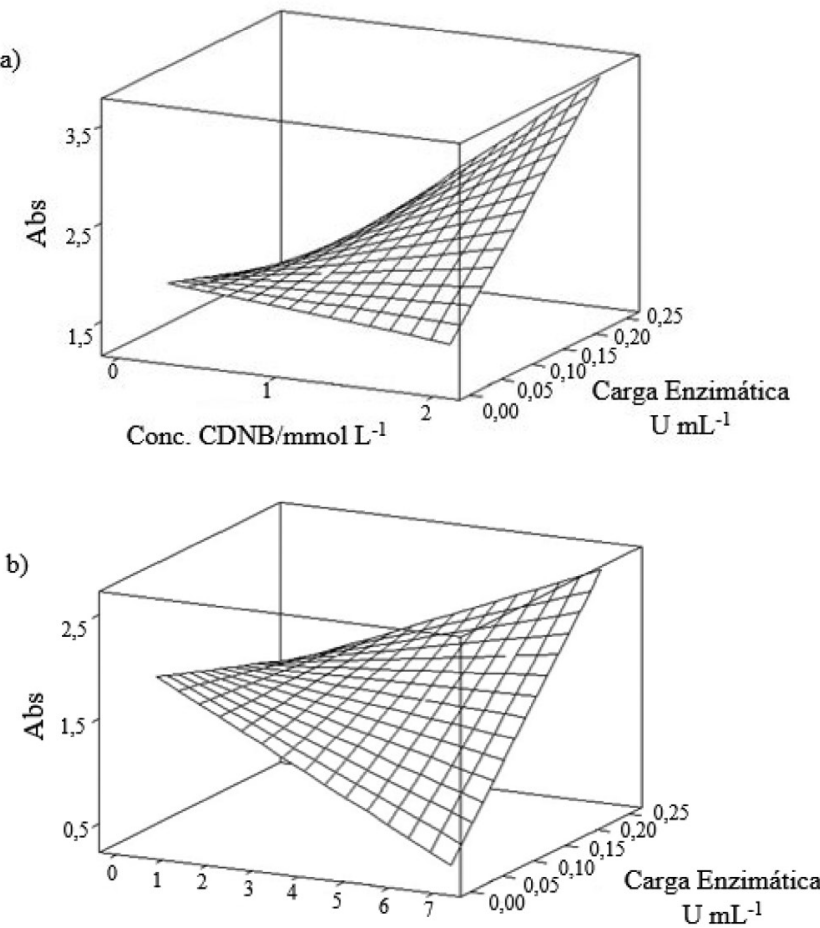

Conc. GSH/mmol L-1

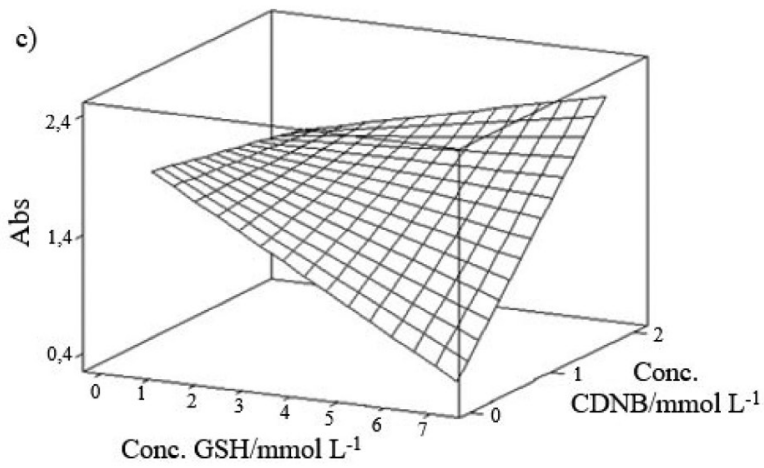

Figura 3. Gráficos de superfície de resposta: (a) com o fator GSH fixo $\left(0,5 \mathrm{mmol} \mathrm{L}^{-1}\right)$, relacionando os fatores concentração de CDNB e carga da enzima GST no sistema; (b) com o fator CDNB fixo $\left(0,2 \mathrm{mmol} \mathrm{L}^{-1}\right)$ relacionando os fatores concentração de GSH e carga da enzima e (c) com o fator carga da enzima fixo $\left(0,0125 \mathrm{U} \mathrm{mL}^{-1}\right)$, relacionando os fatores GSH e CDNB

A Figura $3 b$ indicou claramente que, quando a concentração de CDNB for fixada em $0,2 \mathrm{mmol} \mathrm{L}^{-1}$, é melhor trabalhar com cargas 
enzimáticas mais baixas e concentrações mais elevadas de GSH, para se ter valores de absorbância mais próximos de 0,3 e 0,4 . Na Figura 3c, pode-se verificar que, fixando-se a carga da enzima em $0,0125 \mathrm{U} \mathrm{mL}^{-1}$, a melhor condição ocorreu com a concentração de GSH em nível mais elevado e de CDNB em nível mais baixo.

A partir dos dados obtidos com o planejamento fatorial, e considerando-se o alto custo da enzima optou-se pela configuração: GSH 7,0 $\mathrm{mmol} \mathrm{L}^{-1}$; CDNB $0,2 \mathrm{mmol} \mathrm{L}^{-1}$ e enzima GST $0,0125 \mathrm{U} \mathrm{mL}^{-1}$, como melhor condição experimental a ser adotada para os ensaios cinéticos seguintes, uma vez que será utilizada a menor carga enzimática.

\section{Cinética enzimática na ausência dos inseticidas piretroides}

A glutationa é um tripeptídeo formado pelos aminoácidos: ácido glutâmico, glicina e cisteína que existe no organismo na forma reduzida (GSH) e oxidada (GSSG), sendo que o local ativo responsável por suas propriedades bioquímicas é o grupo tiol da cisteína. ${ }^{20} \mathrm{Na}$ reação, a glutationa S-transferase catalisa a desprotonação da glutationa reduzida gerando o ânion tiolato GS ${ }^{-}$, que possui maior reatividade. A reação de conjugação do tiolato com o CDNB gera um complexo instável e na sequência a formação do conjugado glutationil-dinitrobenzeno (GS-DNB) (Figura 4). ${ }^{21}$

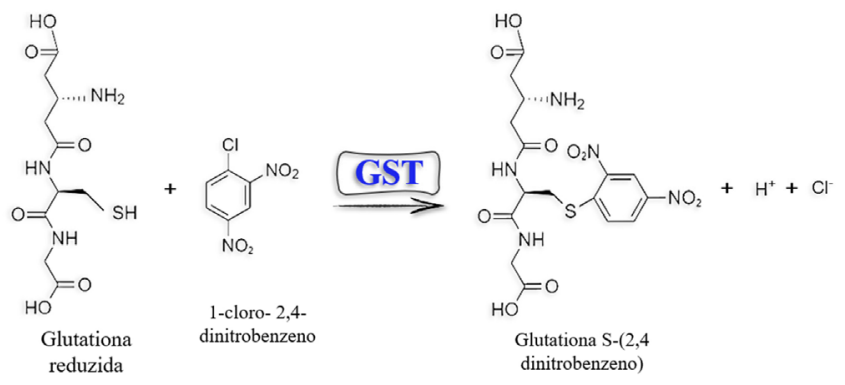

Figura 4. Reação entre 1-cloro-2,4-dinitrobenzeno (CDNB) e glutationa reduzida (GSH), catalisada pela enzima glutationa S-transferase (GST)

Os registros referentes ao comportamento cinético dessa enzima frente a variação das concentrações dos substratos glutationa reduzida-GSH e 1-chloro-2,4-dinitrobenzeno-CDNB encontram-se na Figura 5, em que foi relacionado o inverso da velocidade (1/V), pelo inverso da concentração do substrato $(1 /[\mathrm{S}])$.

A partir da equação dos gráficos de regressão linear: a) $y=258355 x+158901$ com $R^{2}=0,97$ e b) $y=20665 x+58694$ com $\mathrm{R}^{2}=0,98$, os parâmetros de velocidade máxima $\left(\mathrm{V}_{\max }\right)$ e a constante de Michaelis-Menten $\left(\mathrm{K}_{\mathrm{M}}\right)$, apresentados na Tabela 3, foram determinados a fim de avaliar a medida da afinidade da enzima pelo substrato na formação do complexo enzima-substrato.

Os resultados demonstraram que a $\mathrm{V}_{\max }$ mais elevada foi obtida pela reação com concentrações crescentes de CDNB, a qual apresentou um menor valor de $\mathrm{K}_{\mathrm{M}}$, indicando a maior afinidade enzimática. Em um estudo eletroquímico foram encontrados os parâmetros cinéticos da GST, para diferentes concentrações de GSH e CDNB incubados por dois períodos de tempo ( 15 e $30 \mathrm{~min}$ ) e essa enzima demonstrou aproximada afinidade para ambos os substratos (GSH e CDNB) visto que encontrou o valor de $\mathrm{K}_{\mathrm{M}}$ entre $100 \mathrm{e}$ $111 \mu \mathrm{mol} \mathrm{L}{ }^{-1}$ para o CDNB e para GSH entre 104 e $100 \mu \mathrm{mol} \mathrm{L}{ }^{-1} .^{21}$ Em outro estudo realizado com a enzima glutationa S-transferase comercial de fígado equino, foi encontrado menor valor de $\mathrm{K}_{\mathrm{M}}$ para a GSH $\left(0,083 \mathrm{mmol} \mathrm{L}^{-1}\right)$ em relação ao $\operatorname{CDNB}\left(0,15 \mathrm{mmol} \mathrm{L}^{-1}\right){ }^{22}$ Destaca-se que os dados do presente estudo foram registrados por meio de medidas espectrofotométricas e os estudos comparativos foram realizados medindo-se resposta eletroquímica.
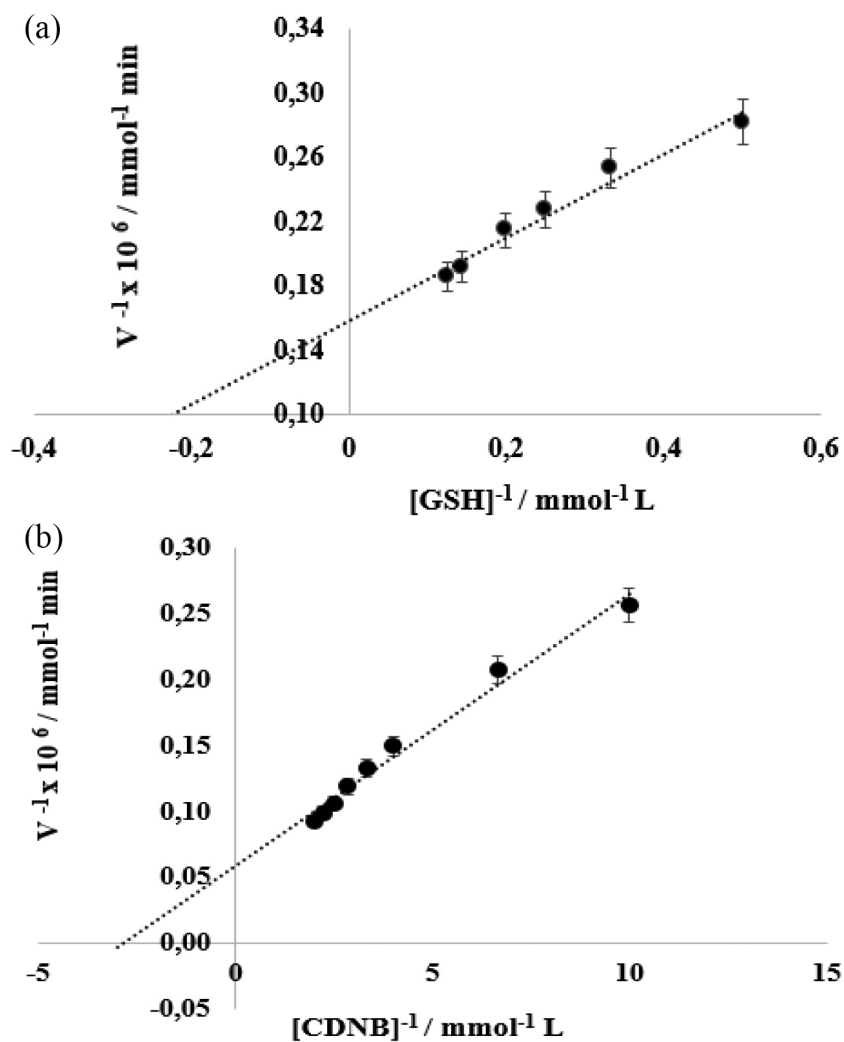

Figura 5. Plotagem do inverso da velocidade pelo inverso da concentração de (a) GSH e (b) CDNB conforme método Lineweaver-Burk

Tabela 3. Parâmetros cinéticos de GST em relação ao CDNB e a GSH

\begin{tabular}{lcc}
\hline Parâmetros & \multicolumn{2}{c}{ Resultados } \\
\cline { 2 - 3 } & CDNB & GSH \\
\hline $\mathrm{V}_{\max }\left(\mu \mathrm{mol} \mathrm{min}^{-1}\right)$ & 0,017 & 0,0063 \\
$\mathrm{~K}_{\mathrm{M}}\left(\mu \mathrm{mol} \mathrm{L}^{-1}\right)$ & 350 & 1620 \\
\hline
\end{tabular}

Em análise, com essa mesma enzima purificada de fígado de ratos, com o substrato $\mathrm{CDNB}$ os autores encontraram $\mathrm{V}_{\text {max }}$ de $0,016 \mathrm{mmol} \mathrm{min}^{-1}$ e o $\mathrm{K}_{\mathrm{M}}$ de $0,323 \mathrm{mmol} \mathrm{L}^{-1}$ espectrofotometricamente. ${ }^{23}$ A diferença verificada entre os valores dos parâmetros cinéticos obtidos no presente estudo que utilizou enzima comercial de fígado equino e os encontrados na literatura, pode estar relacionada ao tipo de técnica analítica aplicada e ao tipo da enzima. Os autores esclarecem que a variação dos valores pode ser dependente da enzima purificada, tendo em vista que também encontraram diferença de valores em seus estudos, onde utilizou-se a GST extraída de cérebro bovino. ${ }^{23}$

Considerando o menor valor de $\mathrm{K}_{\mathrm{M}}$ obtido, pode-se afirmar que o CDNB se mostrou o melhor substrato para a enzima GST. Esse resultado está de acordo com o que foi mostrado anteriormente pelo gráfico de Pareto na Figura 2. Os seguintes ensaios cinéticos, com a enzima GST, foram realizados na presença dos inibidores piretroides.

\section{Cinética de inibição por inseticidas piretroides}

Os resultados obtidos com os ensaios cinéticos contendo variadas concentrações de CDNB, concentrações fixas de GSH e dos quatro pesticidas piretroides estudados, e carga enzimática fixa, estão apresentados nos gráficos duplo-recíproco de Lineweaver-Burk, cujos valores de $\mathrm{R}^{2}$ foram de 0,98 (deltametrina) a 0,99 (aletrina, cipermetrina e fenpropatrina) (Figura 6). 

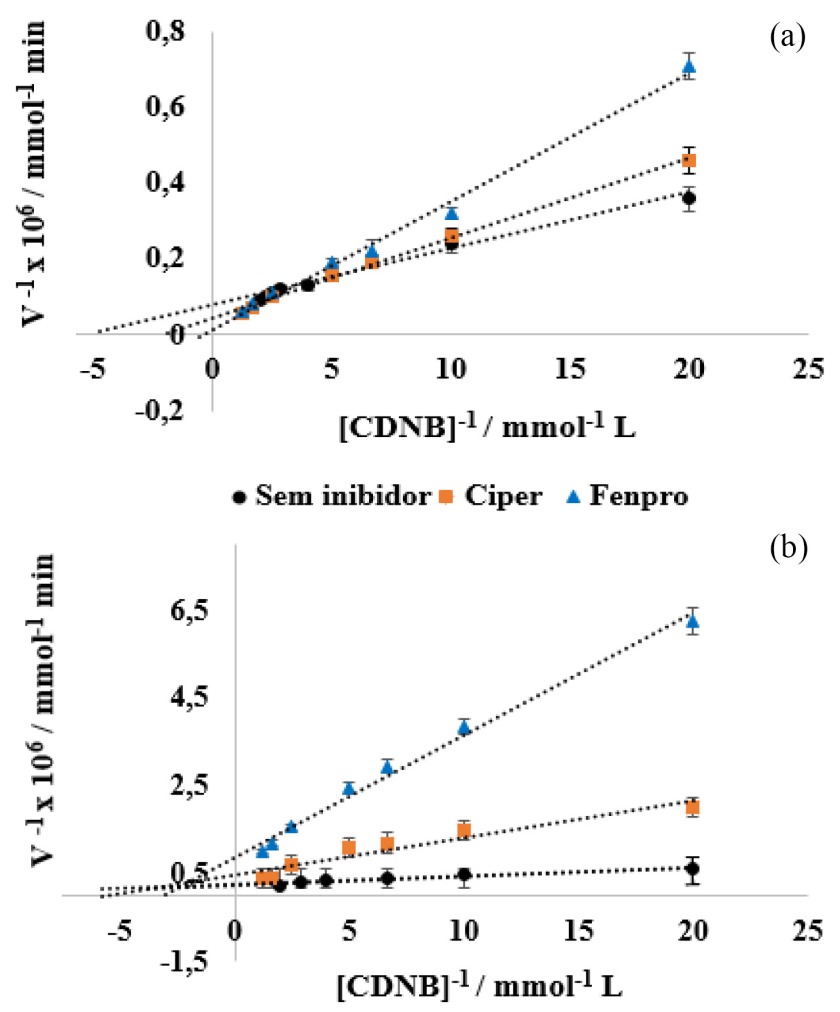

- Sem inibidor Ale $\triangle$ Delta

Figura 6. Representação de Lineweaver-Burk mostrando o efeito da concentração de 0,25 $\mathrm{mg} \mathrm{L}^{-1}$ para cada inibidor (pesticida): (a) cipermetrina e fenpropatrina; (b) aletrina e deltametrina na reação cinética de GSH-CDNB catalisada por GST

A partir dos gráficos de Lineweaver-Burk, foi possível calcular a constante de inibição- $\mathrm{K}_{\mathrm{i}}$ e determinar o tipo de inibição enzimática (Tabela 4). Os estudos in vitro mostraram que a atividade da enzima foi inibida por todos os pesticidas piretroides, com valor de $\mathrm{K}_{\mathrm{i}}$ variando de 0,12 a $20 \mu \mathrm{mol} \mathrm{L}{ }^{-1}$ apresentando a seguinte ordem de inibição do maior para o menor valor $\mathrm{K}_{\mathrm{i}}$ : deltametrina $>$ cipermetrina $>$ aletrina $>$ fenpropatrina. Esse resultado indica que o maior efeito inibitório sobre a GST foi apresentado pelo composto deltametrina. A constante de inibição- $\mathrm{K}_{\mathrm{i}}$ reflete a interação potencial entre a enzima e o inibidor. ${ }^{22}$

No trabalho realizado com o composto deltametrina, os valores de $\mathrm{K}_{\mathrm{i}}$ registrados foram inferiores aos do presente estudo, sendo $5,61 \pm 0,32 \mu \mathrm{mol} \mathrm{L}^{-1}$ utilizando concentração fixa de CDNB e variando a concentração de GSH e 7,96 $\pm 0,97 \mu \mathrm{mol} \mathrm{L}^{-1} \mathrm{em}$ concentração fixa de GSH, variando CDNB, utilizando GST de placenta humana. ${ }^{24} \mathrm{Em}$ outra análise, foi utilizada GST purificada de fígado de peru, GSH como substrato principal, e encontrou-se $0,22 \pm 0,118 \mathrm{mmol} \mathrm{L}^{-1}$ para o piretroide cipermetrina, ${ }^{25}$ valor esse mais elevado em comparação ao registrado na presente pesquisa que utilizou CDNB como substrato. Pode-se inferir que tais variações nos valores de $\mathrm{K}_{\mathrm{i}}$ estejam relacionadas aos diferentes tipos de isoenzimas de GST usadas nos experimentos.

Os valores das constantes de inibição foram submetidos à análise de variância e as médias, foram comparadas pelo teste $t$ de Student não pareado considerando $\mathrm{p}<0,05$. Os resultados mostraram diferença significativa para dois dos compostos piretroides, conforme indicado na Tabela 4.

Dentre os inibidores avaliados, os compostos cipermetrina e fenpropatrina inibiram competitivamente a enzima, ou seja, competiram diretamente com o substrato pelo sítio de ligação da GST. Os demais apresentaram inibição não competitiva (inibição mista) indicando que os inibidores se ligaram tanto à enzima quanto ao complexo enzima-substrato. Esses diferentes comportamentos de inibição podem estar relacionados com os mecanismos de reversibilidade apresentados por cada tipo de composto inibidor. Alguns inibidores enzimáticos são substâncias que possuem estrutura parecida com as dos substratos enzimáticos, mas que não reagem ou reagem lentamente com a enzima; outros afetam a atividade catalítica sem interferir na ligação dos substratos; enquanto outros podem realizar ambos os processos. ${ }^{26}$

Quanto aos resultados de $\mathrm{IC}_{50}$, estão demonstrados na Tabela 4, em que se pode observar que o composto deltametrina se apresentou aproximadamente 3,3 vezes menor que a aletrina com $\mathrm{IC}_{50}$ de 4,3. Esse valor mostra que o piretroide deltametrina reduziu a atividade da enzima GST pela metade, mesmo estando em concentração mais baixa. Esse resultado apontou sua forte capacidade inibitória em relação aos demais pesticidas analisados. Corroborando esse resultado, a literatura relata que, entre os piretroides, a deltametrina foi o composto químico originado da inclusão do grupo substituinte $\alpha$-ciano no grupo 3 -fenoxibenzila, atribuindo maior potência inseticida em relação a outros compostos piretroides previamente sintetizados, como a permetrina. ${ }^{4}$

É importante destacar que os piretroides são amplamente utilizados desde $1970^{27}$ e se tornaram uma preocupação pública por causa da contaminação ambiental e os efeitos tóxicos sobre grupos de animais não alvo, os quais desfavorecem a biodiversidade e o equilíbrio ambiental. ${ }^{28,29}$

Muitos compostos piretroides se apresentam como uma mistura complexa de isômeros com dois ou mais carbonos assimétricos, e sua composição pode ser um importante parâmetro toxicológico e ecotoxicológico, pois normalmente a atividade química e o destino ambiental podem diferir de acordo com o isômero considerado. ${ }^{30,31}$

\section{Contaminação aquática por pesticidas piretroides}

Saber o comportamento dos pesticidas no ambiente é imprescindível para predizer o risco de contaminação ambiental. Assim, dependendo de suas características os pesticidas podem permanecer em diferentes compartimentos do ambiente, tais como atmosfera, solo, água de superfície e subterrânea. ${ }^{32}$ Tendo em vista essa avaliação, encontram-se demonstradas na Tabela 5 as características físico-químicas dos inseticidas piretroides analisados.

Tabela 4. Equação linear, valores de $\mathrm{K}_{\mathrm{i}}, \mathrm{IC}_{50}$ e tipo de inibição sobre a GST

\begin{tabular}{lcccc}
\hline Composto piretroide & Equação linear & $\mathrm{K}_{\mathrm{i}}\left(\mu \mathrm{mol} \mathrm{L} \mathrm{L}^{-1}\right)$ & $\mathrm{IC}_{50}\left(\mathrm{mg} \mathrm{L}^{-1}\right)$ & Tipo de inibição \\
\hline Aletrina & $\mathrm{y}=13579 \mathrm{x}+17508$ & $0,68^{\mathrm{a}} \pm 0,10$ & $4,3 \pm 0,49$ & Não competitiva \\
Cipermetrina & $\mathrm{y}=22074 \mathrm{x}+39488$ & $1,00^{\mathrm{a}} \pm 0,31$ & $2,9 \pm 0,20$ & Competitiva \\
Deltametrina & $\mathrm{y}=283067 \mathrm{x}+804505$ & $20,0^{\mathrm{b}} \pm 0,72$ & $1,3 \pm 0,22$ & Não competitiva \\
Fenpropatrina & $\mathrm{y}=33903 \mathrm{x}+13584$ & $0,12^{\mathrm{c}} \pm 0,02$ & $2,5 \pm 0,17$ & Competitiva \\
\hline
\end{tabular}

$\mathrm{K}_{\mathrm{i}}=$ constante de inibição, calculada a partir da representação de Lineweaver-Burk, na presença do inibidor. Valores de $\mathrm{K}_{\mathrm{i}}$ com mesma letra, na vertical, não diferem significativamente entre si, no nível de $95 \%$ de probabilidade, pelo teste $t$ de Student não pareado. $\mathrm{IC}_{50}=$ concentração de inibidor necessária para reduzir a atividade enzimática em $50 \%$. 
Tabela 5. Características físico-químicas dos inseticidas piretroides estudados

\begin{tabular}{|c|c|c|c|c|c|}
\hline Composto piretroide & $\begin{array}{c}\mathrm{S}_{\mathrm{W}} \\
\mathrm{mg} \mathrm{L}^{-1}\end{array}$ & $\begin{array}{c}\mathrm{DT}_{50} \\
\text { solo (dias) }\end{array}$ & $\begin{array}{c}\mathrm{DT}_{50} \\
\text { água (dias) }\end{array}$ & $\begin{array}{c}\mathrm{K}_{\mathrm{oc}} \\
\mathrm{mL} \mathrm{g}^{-1}\end{array}$ & $\log \mathrm{K}_{\mathrm{ow}}$ \\
\hline Aletrina & $0,0001(\mathrm{~B})$ & 60 (MP) & - & $1400(\mathrm{LM})$ & 4,96 (A) \\
\hline Cipermetrina & $0,009(\mathrm{~B})$ & $21,9(\mathrm{NP})$ & 3 (MR) & 307558 (NM) & $5,55(\mathrm{~A})$ \\
\hline Deltametrina & 0,0002 (B) & 21 (NP) & $17(\mathrm{~L})$ & $10240000(\mathrm{NM})$ & $4,6(\mathrm{~A})$ \\
\hline Fenpropatrina & 0,33 (B) & 28 (NP) & 1 (MR) & $5000(\mathrm{NM})$ & $6,04(\mathrm{~A})$ \\
\hline
\end{tabular}

$\mathrm{NP}=$ não persistente; $\mathrm{MP}=$ moderadamente persistente; $\mathrm{L}=$ lento; $\mathrm{MR}=$ moderamente rápido; $\mathrm{A}=$ alto; $\mathrm{B}=$ baixo; $\mathrm{LM}=$ ligeiramente móvel; $\mathrm{NM}=$ não móvel; (-) = Dado indisponível. Fonte: elaborado a partir de PPDB - Pesticide Properties Database.

Pode-se observar que os compostos analisados apresentam baixa solubilidade em água $(\mathrm{Sw})$, indicando que podem sofrer lenta remoção através de águas pluviais e pouca capacidade de migrarem no ambiente. Esse atributo fisico-químico se refere à probabilidade dos contaminantes serem carreados superficialmente no solo e atingirem as águas superficiais. ${ }^{32}$

Os altos valores para os coeficientes de partição octanol-água $\left(\mathrm{K}_{\mathrm{ow}}\right)$ registrados, sinalizam a tendência bioacumulativa dos quatro compostos piretroides, nos tecidos dos seres vivos. Esse parâmetro determina o caráter lipofílico da molécula do contaminante, ou seja, a afinidade por fases orgânicas, como as membranas biológicas. ${ }^{33}$

Quanto ao tempo de meia vida $\left(\mathrm{DT}_{50}\right)$, apenas para um ingrediente ativo (aletrina) mostrou-se moderadamente persistente no solo, enquanto, para a água, este dado não estava disponível. Os demais inseticidas piretroides não indicaram persistência no solo. O tempo de permanência na água apresentou-se como moderadamente rápido para dois dos piretroides (cipermetrina e fenpropatrina) e lento para um deles (deltametrina).

Os valores do coeficiente de adsorção a matéria orgânica $\left(\mathrm{K}_{\mathrm{oc}}\right)$ mostram que os pesticidas analisados não apresentam mobilidade ou são apenas ligeiramente móveis no solo. Medidas altas para o $\mathrm{K}_{\mathrm{oc}}$ indicam que os contaminantes se fixam com firmeza à matéria orgânica do solo, podendo ser transportados através de partículas de sedimento, e não pela água. ${ }^{34}$

Assim, conforme os critérios analisados, os resultados para o método de GOSS (Tabela 6) mostraram a possibilidade de contaminação de águas superficiais (rios, lagos e lagoas) por compostos piretroides. Os valores indicaram médio potencial de transporte em associação ao sedimento em suspensão e baixo para o transporte dissolvido em água, para três dos pesticidas (cipermetrina, deltametrina e fenpropatrina). Apenas para um composto, aletrina, foi observada alta potencialidade de chegar às águas de superfície através do transporte associado ao sedimento, e média potencialidade, dissolvido em água.

Em ambas as formas, em maior ou menor grau, esses pesticidas podem atingir as espécies aquáticas e provocar impactos negativos sobre elas, seja estando retidos no sedimento ou suspensos na coluna de água. Os peixes, por exemplo, podem sofrer alta toxicidade, devido às propriedades lipofílicas, que fazem com que tais contaminantes sejam absorvidos pelas guelras ${ }^{35} \mathrm{e}$, assim, pode ocorrer processo de biomagnificação ao longo da cadeia alimentar.

Uma vez que estejam presentes na água, os principais efeitos sobre os organismos incluem alterações no crescimento, desenvolvimento, reprodução, respostas farmacocinéticas, patologia, bioquímica, fisiologia e comportamento. ${ }^{36}$
A presença de agrotóxicos em mananciais também pode ser prejudicial para os seres humanos, quando a água é utilizada para abastecimento público, tendo em vista que existe a necessidade de tecnologias mais complexas e seguras de tratamento em relação às que normalmente são usadas para obter a potabilidade. $^{37}$

Tabela 6. Potencial de transporte para os inseticidas piretroides de acordo com o Método de GOSS

\begin{tabular}{lcc}
\hline Potencial & $\begin{array}{c}\text { Potencial de transporte } \\
\text { associado ao sedimento }\end{array}$ & $\begin{array}{c}\text { Potencial de transporte } \\
\text { dissolvido em água }\end{array}$ \\
\hline Aletrina & Alto & Médio \\
Cipermetrina & Médio & Baixo \\
Deltametrina & Médio & Baixo \\
Fenpropatrina & Médio & Baixo \\
\hline
\end{tabular}

Fonte: elaborado a partir de PPDB - Pesticide Properties Database.

Os resultados para os índices de GUS (Tabela 7) evidenciaram que os quatro pesticidas piretroides, não apresentam potencial de contaminação para águas subterrâneas, uma vez que não sofrem lixiviação (GUS < 1,8).

A lixiviação ocorre quando a água se infiltra no solo e avança em profundidade, atingindo o lençol freático. ${ }^{38}$ Quanto menor o valor de meia-vida, menos lixiviável o composto se apresenta. E no caso de pesticidas com valores reduzidos de $\mathrm{K}_{\mathrm{oc}}$, a meia vida passa a exercer influência na lixiviação. Quanto maior o valor de $\mathrm{DT}_{50}$, maior será o potencial de contaminação das águas, dado o maior tempo necessário para a degradação do pesticida no solo e sua consequente permanência no ambiente. ${ }^{39}$ Além da adsorção ao solo, $\mathrm{DT}_{50}$ e a solubilidade em água, a tendência de um pesticida ser lixiviado depende de outras condições, como o tipo de solo, presença e preservação da cobertura vegetal, declividade do relevo e incidência de chuvas. ${ }^{40}$ Também podem sofrer degradação pela influência de outros fatores físicos, químicos e biológicos. ${ }^{41}$

\section{CONCLUSÕES}

Os resultados cinéticos através de espectrofotometria indicaram que os quatros inseticidas piretroides afetam a GST, reduzindo a sua atividade catalítica, principalmente o composto deltametrina, que apresentou maior potencial inibitório, o qual foi analisado através dos valores $\mathrm{K}_{\mathrm{i}}$ e $\mathrm{IC}_{50}$, utilizando-se o CDNB como substrato principal. Embora os inseticidas aplicados neste estudo pertençam à mesma

Tabela 7. Potencial de lixiviação dos inseticidas piretroides de acordo com o Índice GUS

\begin{tabular}{lcccc}
\hline Pesticida & Aletrina & Cipermetrina & Deltametrina & Fenpropatrina \\
\hline Índice GUS & 1,52 & $-1,99$ & $-3,98$ & $-0,80$ \\
Análise de GUS & $\mathrm{NL}$ & $\mathrm{NL}$ & $\mathrm{NL}$ & $\mathrm{NL}$ \\
\hline
\end{tabular}

$\mathrm{NL}=$ não sofre lixiviação. Fonte: elaborado a partir de PPDB - Pesticide Properties Database. 
classe, mostraram mecanismo de inibição diferentes dependendo do composto.

A avaliação quanto ao destino no ambiente indicou que os inseticidas podem se fixar principalmente às partículas do solo e sedimento, e através do escoamento superficial podem atingir o meio aquático. Uma vez nesse ambiente, podem se depositar na região de fundo, entrar em contato com as espécies bentônicas e assim promover uma acumulação progressiva através dos níveis tróficos, colocando em risco a saúde humana, seja pela alimentação ou pelo consumo da água.

Os dados apurados com esta pesquisa demonstraram a viabilidade do emprego da enzima glutationa S-transferase como molécula de bioreconhecimento em trabalhos futuros no que tange ao desenvolvimento de biossensores para detecção de pesticidas piretroides.

\section{AGRADECIMENTOS}

Agradecemos à CAPES (Coordenação de Aperfeiçoamento de Pessoal de Nível Superior), à Universidade Federal do Maranhão (UFMA) e ao Núcleo de Análise de Resíduos de Pesticidas (NARP/ UFMA), pelo apoio institucional.

\section{REFERÊNCIAS}

1. Balcı, N.; Türkan, F.; Şakiroğlu, H.; Aygün, A.; Şen, F.; Heliyon 2019, $5, \mathrm{e} 01422$.

2. Aktar, W.; Sengupta, D.; Chowdhury, A.; Interdiscip. Toxicol. 2009, 2, 1.

3. Santos, M. A. T. Dos; Areas, M. A.; Reyes, F. G. R.; Aliment. Nutr. 2007, $18,339$.

4. Soderlund, D. M.; Clark, J. M.; Sheets, L. P.; Mullin, L. S.; Piccirillo, V. J.; Sargent, D.; Stevens, J. T.; Weiner, M. L.; Toxicology 2002, 171, 3.

5. Morgan, M. K.; MacMillan, D. K.; Zehr, D.; Sobus, J. R.; J. Expo. Sci. Environ. Epidemiol. 2018, 28, 40.

6. Gan, J.; Lee, S. J.; Liu, W. P.; Haver, D. L.; Kabashima, J. N.; J. Environ. Qual. 2005, 34, 836.

7. Trevizan, L. R. P.; Baptista, G. C.; Sci. Agric. 2000, 57, 199.

8. Mello, I. N. K.; Silveira, W. F.; Acta Vet. Bras. 2012, 6, 94.

9. Sánchez-Bayo, F.; Goka, K.; Hayasaka, D.; Front. Environ. Sci. 2016, 4,1 .

10. Islam, S.; Sajib, S. Das; Jui, Z. S.; Arabia, S.; Islam, T.; Sci. Rep. 2019, 1.

11. Cummins, I.; Dixon, D. P.; Freitag-Pohl, S.; Skipsey, M.; Edwards, R.; Drug Metab. Rev. 2011, 43, 266.

12. Kumar, S.; Trivedi, P. K.; Front. Plant Sci. 2018, 9, 1.

13. Borah, H.; Dutta, R. R.; Gogoi, S.; Medhi, T.; Puzari, P.; Anal. Methods 2017, 9, 4044.

14. Carvalho Neta, R. N. F.; Abreu-Silva, A. L.; Lat. Am. J. Aquat. Res. 2013, 41, 217.
15. Ribeiro, E. B.; Bastos, L. S.; Galeno, L. S.; Mendes, R. S.; Garino, F.; Carvalho-Neta, R. N. F.; Costa, F. N.; Mar. Pollut. Bull. 2016, 113, 182.

16. Ribeiro, E. B.; Noleto, K. S.; de Oliveira, S. R. S.; Batista de Jesus, W.; de Sousa Serra, I. M. R.; de Almeida, Z. S.; Andrade, T. S. O. M.; Soares, R. A.; Antonio, Í. G.; Santos, D. M. S.; Jorge, M. B.; Carvalho Neta, R. N. F.; Mar. Pollut. Bull. 2020, 158, 111348.

17. Habig, W. H.; Pabst, M. J.; Jakoby, W. B.; J. Biol. Chem. 1974, 249, 7130.

18. Goss, D. W.; Weed Sci. Soc. Am. 1992, 6, 701.

19. Gustafson, D. I.; Environ. Toxicol. Chem. 1989, 8, 339.

20. Rover Júnior, L.; Höehr, N. F.; Vellasco, A. P.; Kubota, L. T.; Quim. Nova 2001, 24, 112.

21. Enache, T. A.; Oliveira-Brett, A. M.; Bioelectrochemistry 2015, 101, 46.

22. Borah, H.; Gogoi, S.; Kalita, S.; Puzari, P.; J. Electroanal. Chem. 2018, $828,116$.

23. Torres, M. C. L.; Soares, N. de F. F.; Maia, J. F.; Ciência e Tecnol. Aliment. 2004, 24, 243.

24. Markus, V.; Teralı, K.; Dalmizrak, O.; Ozer, N.; Environ. Toxicol. Pharmacol. 2018, 61, 18.

25. Güller, P.; Akkemik, E.; Kör, S.; Çiftçi, M.; J. Inst. Sci. Technol. 2018, $8,211$.

26. Voet, D.; Voet, J. G.; Bioquímica; 4th ed.; Artmed editora: Porto Alegre, 2013.

27. Montanha, F. P.; Pimpão, C. T.; Revista Científica Eletrônica de Medicina Veterinária 2012, 9, 58.

28. Chen, S.; Chang, C.; Deng, Y.; An, S.; Dong, Y. H.; Zhou, J.; Hu, M.; Zhong, G.; Zhang, L.-H.; J. Agric. Food Chem. 2014, 62, 2147.

29. Xiong, J.; Zhang, X.; Huang, J.; Chen, C.; Chen, Z.; Liu, L.; Zhang, G.; Yang, J.; Zhang, Z.; Zhang, Z.; Lin, Z.; Xiong, N.; Wang, T.; Mol. Neurobiol. 2016, 53, 995.

30. Ye, J.; Zhao, M.; Liu, J.; Liu, W.; Environ. Pollut. 2010, 158, 2371.

31. Parente, C. E. T.; Azevedo-Silva, C. E.; Meire, R. O.; Malm, O.; Orbital 2018, 10, 337.

32. Cabrera, L.; Costa, F. P.; Primel, E. G.; Quim. Nova 2008, 31, 1982.

33. Milhome, M. A. L.; de Sousa, D. de O. B.; Lima, F. de A. F.; Nascimento, R. F. do; Eng. Sanit. e Ambient. 2009, 14, 363.

34. Guarda, P. M.; Pontes, A. M. S.; Domiciano, R. de S.; Gualberto, L. da S.; Guarda, E. A.; Desafios - Revista Interdisciplinar da Universidade Federal do Tocantins 2020, 7, 123.

35. Rehman, H.; Aziz, A. T.; Saggu, S.; Abbas, Z. K.; Mohan, A.; Ansari, A. A.; Journal of Entomology and Zoology Studies 2014, 2, 60.

36. Oliveira, G. A. V.; Silva, J. M. S. F.; Quim. Nova 2017, 40, 726.

37. Fernandes Neto, M. L.; Sarcinelli, P. N.; Eng. Sanit. Ambiental 2009, $14,69$.

38. Rebelo, R. M.; Caldas, E. D.; Quim. Nova 2014, 37, 1199.

39. Ferracini, V. L.; Pessoa, M. C. Y. P.; Silva, A. S.; Spadotto, C. A.; Pesticidas: Revista de Ecotoxicologia e Meio Ambiente 2001, 11, 1.

40. Dellamatrice, P. M.; Monteiro, R. T. R.; Revista Brasileira de Engenharia Agrícola e Ambiental 2014, 18, 1296.

41. Vryzas, Z.; Curr. Opin. Environ. Sci. Heal. 2018, 4, 5. 